\title{
Editorial
}

\section{Adapting to climate change}

Q xpectations that a reduction in greenhouse gases could be as easily agreed and implemented as was the Montreal Protocol for CFCs have proved sadly wrong. It is now clear that any serious global reduction in these gases will only come - if at all - too late to be of any use in limiting the projected impacts. Despite the efforts of the IPCC the forces of self-interest in both politics and business are too powerful for rational science to overcome, and we will be lucky to contain the temperature rise even to $4^{\circ} \mathrm{C}$.

So, if Kyoto has failed, what is the next step? It must be the development of programmes for adaptation, an approach that many governments seemed to want to avoid for as long as possible. Others saw it differently, recognizing that at best Kyoto was only a very small step which would make little practical difference. Adaptation always seemed to be the much more pragmatic option as it recognizes the role of the individual, shows how the interactions of change affect our lives in many complex ways whether we are in China or the Maldives, and, since it is an incremental process, does not require immediate and dramatic changes to lifestyles.

But what do we need to modify, how much and when? One view certainly is that the pervasive nature of the problem needs adaptation to underlie virtually all important future decisions. From water usage to town planning, transport to health, energy to leisure - all need to take climate change into account. You might expect that such wide-ranging imperatives would require governments to ensure that, for example, infrastructure investment was checked against future environments, that water pricing was reassessed, that building regulations were changed, and so on. And there are indications that this is happening in at least some countries but real international leadership here seems lacking. The United States, as the second largest polluter but also a major innovator, should be out in front but instead is plagued by sceptics, self-interest and the world's largest debts. Institutionalised lobbying and political patronage seems to have usurped the ability of American legislators to act in the interests of the common good so little can be expected from them. On this side of the Atlantic the European Union is mired in debt, unemployment and arguments over fiscal and political vision, so there seems little reason to be optimistic about them leading planning for change on a co-ordinated basis.

Any hope lies elsewhere, with initiatives in individual nation states. A report in 2009 provided an initial comparison of adaptive strategies in the UK, Germany, France, Denmark, Finland, Sweden, Netherlands, Latvia, Portugal, and Spain, which seemed quite encouraging (http://www.peer.eu/ fileadmin/user_upload/publications/PEER_Report1.pdf). There are serious cross-departmental attempts to look at practical advice for coping with threats like flooding, water shortage, energy saving, heat stress and so on, highlighting practical objectives but with varying national emphases. Crucial to any scheme for adaptation is a long-term view over several generations - it is not just the present politicians who need educating but also the children within the educational system who must see adapting to change as a fundamental part of their future. And that initiative seems yet to be adopted as a high priority.

But clever adaptation alone will not be enough to save us. Diminishing environmental capital, an increasingly unpredictable climate and too many people are a disastrous mixture. With the global population at 7 billion where is the global initiative on population control that will make adaptation work? Are we really doomed by our uncontrolled breeding as Paul Erlich has suggested?

D.W.H. WALTON 